\title{
The Influence of Magnetic Hysteresis in Magnetic Circuit Sheets on Transient Processes in Electrical Machines
}

\author{
Nenad A. Marković \\ Kosovo and Metohija Academy of Applied Studies, Department Urosevac - Leposavic, Serbia \\ Email: nen.mark74@yahoo.com
}

\author{
Slobodan N. Bjelić \\ Faculty of Technical Sciences, Kosovska Mitrovica, Serbia \\ Email: slobodanbjelic49@yahoo.com
}

\author{
Filip N. Marković \\ Faculty of Technical Sciences, Kosovska Mitrovica, Serbia \\ Email: ridjikm@gmail.com
}

Received: 21 March 2021; Accepted: 03 May 2021; Published: 08 June 2021

\begin{abstract}
The character of transient processes in electrical machines and transformers defines the shape of magnetization curve of the magnetic circuit, i.e. of its sheets. Approximate analytical or numerical methods are used to determine the influence of saturation and hysteresis on transient processes. This paper presents an analytical method for the calculation of transient process in a magnetic circuit with assumed magnetization characteristic, one part of which contains saturation. An operator calculus was used to solve Maxwell's equations that characterize the transient process. The applied method has been verified by the simulation results using the adapted part of psbxfosaturable.mdl of the MATLAB Simulink software package. It is also shown that due to saturation and influence of hysteresis, additional free components appear in the sheets of the magnetic circuit (recognized in obtained values of current, induction and flux). Analysis of the shape of time diagrams of quantities also shows that the time constants of these quantities increase. In the linear part of the magnetization curve, solutions with higher accuracy are obtained, and in the part of saturation in which the accuracy of the method was not in foreground, only a qualitative analysis of the transient process has been achieved. In comparison with other methods, two regimes of transient processes when given are analyzed: magnetic excitation forces and field strengths, and magnetic fluxes and inductions.
\end{abstract}

Index Terms: Electrical Machines, Magnetic Circuit, Sheets, Transient Process, MATLAB Simulink

\section{Introduction}

The transformer operates in normal mode and disturbance mode, which includes faults with extreme deviations of electrical sizes and disturbances with sizes that are close to normal in values. In reality, electrical quantities are predominantly non-sinusoidal due to the presence of nonlinear components and loads in power systems [1]. The aim of this paper is to theoretically determine the influence of hysteresis saturation on the appearance of free components of current, magnetic induction and flux in magnetic circuit sheets. Limitations in the research are the geometrical shapes of the conducting parts of the windings of the primary and secondary elements of magnetic circuits. As shown in the paper, this can be overcomed by the correct choice of coordinate systems in which the elements of electrical and magnetic circuits are located.

In transient processes of electric machines [2], in addition to the fundamental harmonics and higher voltage harmonics, there are also:

- excessive values of currents and magnetic inertia and saturation of magnetic circuits,

- large electrodynamic forces caused by excessive currents and influence of interaction of these currents with the shells and armor of ferromagnetic materials,

- creation of conditions for ferroresonance, mechanical vibrations in core sheets [3-4], 
- accelerated aging of insulation material due to cyclic changes of induction,

- increase in losses in copper (due to fundamental and higher harmonics),

- $\quad$ increased losses in the core (due to hysteresis and vortical currents).

The influence of hysteresis on transient process has been analyzed on a separate sheet as part of a magnetic circuit containing a package of rectangular sheets of geometric dimensions $x>\Delta, l>\Delta$. The task of a magnetic circuit is to spatially distribute the magnetic excitation forces or magnetic fluxes that produce currents in electric circuits. The shape and dimensions of the sheets in magnetic circuit allow the propagation of plane electromagnetic (EM) waves and influence of vortical currents is compensated in each alternative middle sheet between two adjacent sheets [5-7]. The analysis of the influence of magnetic hysteresis includes only one sheet.

The plane polarized wave corresponds to the EM field of alternating current whose vectors of electric $E$ and magnetic $H$ field strength is functions of only one coordinate of space, for ex. $z$. At each point of time they are positioned in a plane normal to the direction of propagation and their modules, direction and rotors are constant in the plane. In contrast to this abstract wave, an unpolarized or natural plane wave corresponds to a field whose vectors $E$ and $H$ they also lie in a plane normal to the direction of propagation, but their modules, direction, and rotors depend on the coordinates in the plane. The propagation of unpolarized waves defines the processes in the parts of the power element assemblies in electrical engineering and if the vectors $E$ and $H$ the fields of the sine function of the time wave are called monochromatic [8-10]. Computer simulation today is one of the leading methods for solving, describing, understanding, and analyzing transient processes in electrical machines.

Therefore, in this paper, based on the known procedures that apply to transient processes in electrical machines, the relations related to magnetic hysteresis are determined, and validity of obtained expressions is confirmed by simulation.

The paper is organized as follows: Section 2 provides an overview of the literature of related papers in the field of transient processes in electrical machines related to the definition of sheets as part of magnetic circuits. In Section 3 a mathematical model is given by which the analytical equations for the assumed magnetization curve and characteristics of magnetic circuit sheets are derived. Section 4 gives simulation results and discussion of the influence of saturation effect on values of currents and voltages in transformer primary in transient process. Finally, some concluding considerations are presented in Section 5.

\section{Literature Review}

The theory of single-phase and three-phase transformers was developed in the first half of the XX century and Steinmetz, Richter, Kron, Fitzgerald [9], AI Voljdek [11], are just some of the names of authors who dealt with magnetic circuits of transformers.

In the works of Match [12] and Chapman [13], normal stationary states with one frequency and classical transformer parameters are analyzed. In the available literature, in addition to the basic ones, higher harmonics are also taken into account in the analysis of normal and transient states [14-15].

Although the windings of current, energy and voltage transformers are sets of electrical circuits composed of a large number of elementary winding circuits, ie. circuits with distributed parameters, models are mainly formed with electric circuits with distributed parameters. Small transformers, in terms of power and geometric dimensions, are no exception to this rule. The analysis must include the influence of the parameters of linear self-inductance of the conductor (along the conductor), capacitance between the previous and next winding, reactions caused by mutual induction of one part of the winding to another and between primary and secondary, conclude E.F. Fuchs and others [16]. Also, B. Geller and A. Veverka [5] considered pulse overvoltages in transformers and presented the results of experiments for different types of windings and transformer constructions. We must not forget the Fouc currents in the conductors and the magnetic core and the losses caused by hysteresis [16].

Although the authors in references [1-5] and [6-8] proposed a procedure similar to the method used in this paper, the method has not been referenced and verified in detail. Due to the "small" number of references on this topicand based on insights into the mentioned references and other available literature to date, the authors have not found a suitable and sufficiently accurate, theoretical procedure that would adequately determine the unpredictable distribution and spreading of voltage and current waves. For this reason, this paper was created, and the simulation results confirmed that the proposed method and the obtained theoretical model are adequate and functional.

Insight into the mentioned references and other available literature to date, the authors have not found a suitable and sufficiently accurate, theoretical procedure that would adequately determine the unpredictable distribution and propagation of voltage and current waves in the coils.

\section{An advanced version of Mathematical Method of Magnetic Circuit}

According to the definition of EM waves, vectors $E$ and $H$ also lie in the plane (x0y) normal to the direction of propagation-axis $z$, Fig. 1 . So, all $z$ components along the axis are equal to zero: $E_{z}=0, H_{z}=0$. Propagation of an 
unpolarized EM plane wave in the middle, in which it is $\mu=$ const.,$\varepsilon=$ const.,$\sigma=$ const. , describe Maxwell's equations I and II [8] and the solution is obtained by their application in Cartesian coordinate system adapted to the geometric shape of sheets, Fig. 1.a:

$$
\begin{aligned}
& \operatorname{rot} \vec{H}=\vec{J}=\sigma \vec{E} \Leftrightarrow-\frac{\partial H(t, z)}{\partial z}=\sigma E(t, z) \\
& \operatorname{rot} \vec{E}=-\mu \frac{\partial \vec{H}}{\partial t} \Leftrightarrow \frac{\partial E(t, z)}{\partial z}=-\frac{\partial B(t, z)}{\partial t}
\end{aligned}
$$

\section{Where}

$H(t, z)$ is the strength of magnetic field depends on time and coordinate $(t, z)$,

$\sigma$ is the electrical conductivity of sheet metal material,

$E(t, z)$ is the electric field strength depends on time and coordinate $z$,

$B(t, z)$ magnetic induction (normal component of induction that penetrates the sheet metal).

In Fig. 1 shows a magnetic circuit in which the propagation of a plane EM wave is determined.
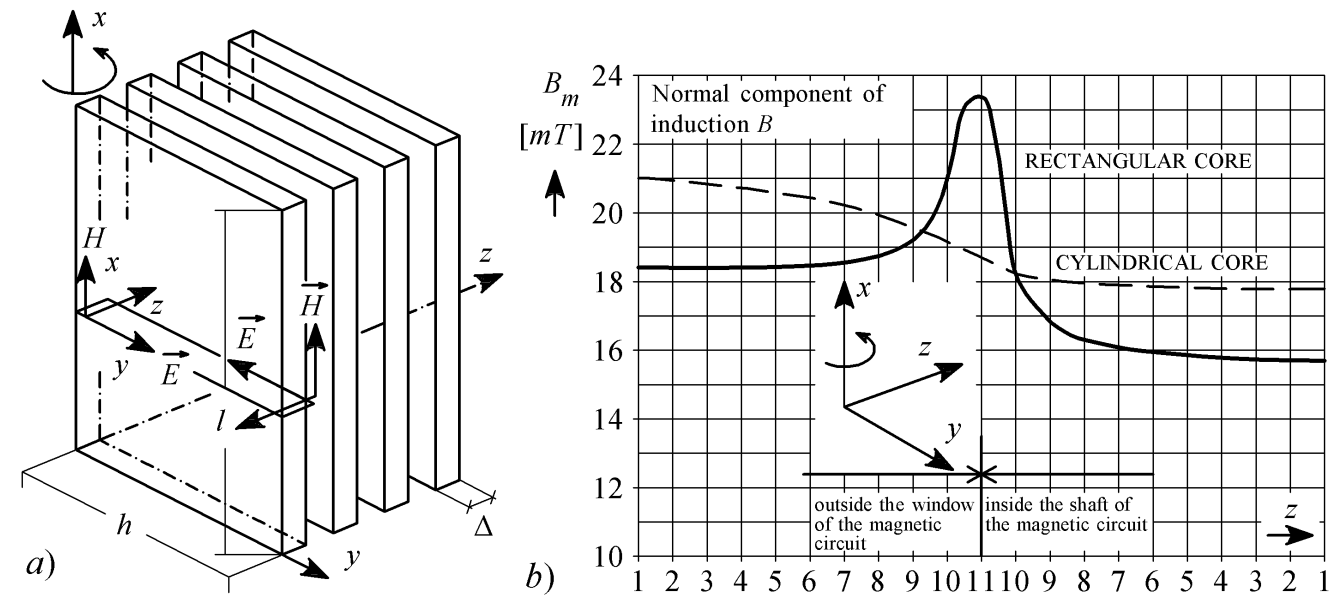

Fig. 1. a) The size and domain of a "sheet-sheet" of a magnetic circuit in which the propagation of a plane EM wave is determined, b) the values of the normal components of induction in the core (column) of a rectangular core (model) and cylindrical core (model) [17]

At industrial frequencies, in transformers, the currents of dielectric displacements can be neglected in relation to the conduction currents because the value $\varepsilon \frac{\partial E}{\partial t}<<\sigma E$.

For a simple periodic plane wave of an electric field which is a consequence of the voltage of an external source $E=E_{m} e^{j \omega t}$ relation of these currents is:

$$
\frac{J_{\text {die.dis. }}}{J_{\sigma}}=\varepsilon \frac{\partial E}{\partial t} \frac{1}{\sigma E}=j \frac{\omega \varepsilon}{\sigma}
$$

For copper approximately:

$$
\left|\frac{J_{\text {die.dis. }}}{J_{\sigma}}\right|=\left|\frac{\omega \varepsilon}{\sigma}\right|=\frac{2 \cdot 3,14 \cdot 50 \cdot 8.85 \cdot 10^{-12}}{58 \cdot 10^{6}} \cong 47,9 \cdot 10^{-18},
$$

For iron approximately:

$$
\left|\frac{J_{\text {die.dis. }}}{J_{\sigma}}\right|=\left|\frac{\omega \varepsilon}{\sigma}\right|=\frac{2 \cdot 3,14 \cdot 50 \cdot 8.85 \cdot 10^{-12}}{8 \cdot 10^{6}} \cong 347,2 \cdot 10^{-18} .
$$


When analyzing a process in a device that has a ferromagnetic circuit with a current dynamic characteristic $B=f(H)$ it is necessary to solve the nonlinear differential equation by analogy with the electric one $R L$ and $R C$ circuit:

$$
R i+N_{n} S \frac{d B}{d t}=u \Leftrightarrow\{u(t) \equiv H(t) i(t) \equiv B(t)\} \Leftrightarrow k_{1} B+k_{2} \frac{\partial B}{\partial t}=H(t)
$$

The current dynamic characteristic depends on the speed and law of change of the magnetic field or flux. If it is $\frac{d B}{d t}=\frac{d B}{d H} \frac{d H}{d t}=\mu_{0} \mu_{d}(H) \frac{d H}{d t}$ then also $B=\mu_{0} \mu H$.

Due to the active electrical losses in the magnetic circuit sheets, the equivalent sinusoids of the vector $E$ and $H$ the field are phase shifted. According to Voljdek's recommendation [11], the influence of these losses is taken into account by introducing complex magnetic permeability (this term was first introduced by Voljdek) which relies on the periodic sinusoidal character of electric $E$ and magnetic $H$ field strength:

$$
\hat{\bar{\mu}}=\hat{\bar{B}} / \hat{\bar{H}}=\bar{\mu}_{r}-j \bar{\mu}_{a}
$$

Where $\bar{\mu}_{r}$ is the conservative part conditioned by the reactive component of the winding impedance, $\bar{\mu}_{a}$ is the consumer (active) part that characterizes the losses in the magnetic circuit.

The excitation of the device's magnetic circuits can be realized in one of two modes:

- when the magnetic excitation force/ampere windings are set (magnetic circuits of current transformers, current limiting reactors, inductors, current relays ...),

- when the magnetic flux/supply voltage is set (magnetic circuits of power transformers, rotating machines, voltage transformers and relays, electromagnets) [18].

Depending on the magnetization regime from equations (1) are determined $E(t, z)$ or $H(t, z)$ and then the current field strength values $H(t)$ and induction $B(t)$. If the changes in the magnitudes of the EM field are sine functions, what are the conditions for the application of a complex value of permeability, and if the magnetization curve and its hysteresis are given, the field strength $H(t, y)$ and induction $B(t, y)$ connect relations for one of the two regimes of transient processes [11], [19], ie. when given:

- magnetic excitation forces and field strengths

$$
H(t, z)=k_{1} B(t, z)+k_{2} \frac{\partial B(t, z)}{\partial t}
$$

- magnetic fluxes and induction

$$
B(t, z)=\dot{k_{1}} H(t, z)+\dot{k_{2}} \int_{0}^{t} H(t, z) d t
$$

Where

$k_{1}=\frac{\cos \varsigma}{\mu}, k_{2}=\frac{1}{\omega} \frac{\sin \varsigma}{\mu}, \hat{k_{1}}=\mu \cdot \cos \varsigma \quad \grave{k_{2}}=\omega \mu \cdot \sin \varsigma$ is the constant,

$\mu$ is the magnetic permeability module (conductivity),

$\varsigma$ is the magnetic induction delay angle according to magnetic field strength (phase position).

It has already been said that equations (5.a and 5.b) are analogous to the expressions $R L$ and $R C$ circuit, and with the introduced value of complex magnetic permeability, transformation from complex to time domain is possible. By applying the Laplace transform and zero initial conditions for the sheet metal domain with the help of the operator $p=j \omega[20]$ and convolution and translation theorems [9-10], [21-23], we get:

$$
\begin{aligned}
& E(t) \doteq E(p), H(t) \doteq H(p), B(t) \doteq B(p), \phi(t) \doteq \phi(p) \\
& E(0)=0, H(0)=0, B(0)=0, \phi(0)=0
\end{aligned}
$$


After the transformation, equations (1) and (5) are reduced to the form:

$$
\begin{aligned}
& \frac{\partial H(p)}{\partial z}=-\sigma E(p), \frac{\partial E(p)}{\partial z}=-p B(p),-\frac{1}{p} \frac{\partial E(p)}{\partial z}=B(p) \\
& H(p)=-B(p)\left[k_{1}+k_{2} p\right], B(p)=H(p)\left[k_{1}+\frac{1}{k_{2}} p\right]
\end{aligned}
$$

By differentiating equations (7) by $H(p)$ and $E(p)$ relations for both regimes are obtained:

$$
\begin{aligned}
& \frac{\partial^{2} H(p)}{\partial z^{2}}-\sigma \frac{p}{k_{1}+p k_{2}} H(p)=0 \\
& \frac{\partial^{2} E(p)}{\partial z^{2}}-\sigma \frac{1}{p k_{1}+k_{2}} E(p)=0
\end{aligned}
$$

Solutions per $H(p)$ and $E(p)$ are complex operator functions:

$$
\begin{aligned}
& H(p)=H_{0}(p) \frac{\operatorname{ch} \lambda_{1} z}{\operatorname{ch} \lambda_{1} \frac{\Delta}{2}}=\frac{F_{1}(p)}{F_{2}(p)} \\
& E(p)=E_{0}(p) \frac{\operatorname{ch} \lambda_{2} z}{\operatorname{sh} \lambda_{2} \frac{\Delta}{2}}=\frac{f_{1}(p)}{f_{2}(p)}
\end{aligned}
$$

Induction values, knowing that $\int \operatorname{shaz}=\frac{1}{a} \operatorname{chaz}, \int \operatorname{chaz}=\frac{1}{a} \operatorname{shaz}$ will be:

$$
\begin{aligned}
& B(p)=H_{0}(p) \frac{\lambda_{1}^{2}}{p \sigma} \frac{\operatorname{ch} \lambda_{1} z}{\operatorname{ch} \lambda_{1} \frac{\Delta}{2}}=\frac{F_{3}(p)}{F_{4}(p)} \\
& B(p)=E_{0}(p) \frac{\lambda_{2}}{p} \frac{\operatorname{sh} \lambda_{2} z}{\operatorname{sh} \lambda_{2} \frac{\Delta}{2}}=\frac{f_{3}(p)}{f_{4}(p)}
\end{aligned}
$$

A complex flux value transformed by an operator $p=j \omega$ which penetrates through a unit of sheet width $\Delta$, is determined from equation (10) which defines the flux:

$$
\begin{aligned}
& \phi(p)=h \int_{-\Delta / 2}^{\Delta / 2} B(p) d z=h \cdot 2 H_{0}(p) \frac{\lambda_{1}}{p \sigma} \operatorname{th} \lambda_{1} \frac{\Delta}{2}=\frac{F_{5}(p)}{F_{6}(p)} \\
& \phi(p)=h \int_{-\Delta / 2}^{\Delta / 2} B(p) d z=h \cdot 2 E_{0}(p) \frac{1}{p}=\frac{f_{5}(p)}{f_{6}(p)}
\end{aligned}
$$

The values of the Laplace transform transformed from the original are: for the electric field strength $E_{0}(p)$, for the strength of the magnetic field $H_{0}(p)$, and the combined EM parameters of the magnetic circuit are: $\lambda_{1}=\sqrt{\frac{p \sigma}{k_{1}+p k_{2}}}$ and $\lambda_{2}=\sqrt{\frac{\sigma}{p k_{1}+k_{2}}}$.

In the mode of given magnetic excitation forces of medium length of the magnetic line $l_{s r}$, strength of the field is: 


$$
H_{0}(p)=\frac{I(p)}{l_{s r}} N, I(p)=\frac{H_{0}(p)}{N} l_{s r}
$$

It is in the mode of known magnetic fluxes:

$$
B(p)=H_{0}(p) \frac{\lambda_{2}^{2}}{p \sigma} \frac{\operatorname{ch} \lambda_{2} z}{\operatorname{ch} \lambda_{2}(\Delta / 2)}=\frac{F_{3}(p)}{F_{4} `(p)}
$$

Flux is calculated using a constant $\lambda_{2}$ and equastion (11) becomes:

$$
\begin{aligned}
& \phi(p)=h \int_{-\Delta / 2}^{\Delta / 2} B(p) d z= \\
& =h \cdot 2 H_{0}(p) \frac{\lambda_{2}}{p \sigma} \operatorname{th} \lambda_{2} \frac{\Delta}{2}=\frac{F_{5}(p)}{F_{6}(p)}
\end{aligned}
$$

In the mode of given fluxes (induction) and $N_{\text {lesf }}$-number of sheet metal of magnetic circuit when the supply voltage of the source is set $v=V_{m} \sin (\omega t-\varphi)$, the strength of the field is:

$$
H_{0}(p)=\frac{\sigma \cdot E_{0}(p)}{\lambda_{2}} \operatorname{cth}\left(\lambda_{2} \frac{\Delta}{2}\right)
$$

The strength of the electric field is determined from Kirchhoff's rule for an electric circuit which results in the expected value of the flux:

$$
V(p)=R I(p)+p \phi(p)=R \frac{H_{0}(p)}{N_{\text {wind }}} l_{s r}+h N_{\text {list }} 2 E_{0}(p)=E_{0}(p)\left[R \frac{\sigma l_{s r}}{\lambda_{2} N} c t h\left(\lambda_{2} \frac{\Delta}{2}\right)+2 h N_{\text {list }}\right]
$$

Where strength of electric field is $E_{0}(p)=\frac{V(p)}{R \frac{\sigma}{\lambda_{2}} \frac{l_{s r}}{N} \operatorname{cth} \lambda_{2} \frac{\Delta}{2}+2 h N_{\text {list }}}$.

Received value $E_{0}(p)$ in a qualitative sense it confirms the correctness of the previous procedure. Original function $H, B$ and $\Phi$ could be determined by applying the decomposition theorem [15], [21].

The originals of the functions are specified below $E(t) \doteq E(p), H(t) \doteq H(p), B(t) \doteq B(p), \phi(t) \doteq \phi(p)$, when the field strength function and the magnetizing current are simple periodic:

$$
H_{0}(p)=H_{m} \frac{\omega \cos \varphi-p \sin \varphi}{p^{2}+\omega^{2}} \therefore H_{m}=\frac{I_{m}}{l_{s r}} N
$$

Where $\varphi$ is the phase position-switching angle.

For such a given excitation, the responses are as follows:

$$
\begin{aligned}
& H=\sum \frac{F_{1}\left(p_{i}\right)}{F_{2}\left(p_{i}\right)} e^{p_{i}{ }^{\grave{ }}}=H_{p p}+H_{s l} \\
& B=\sum \frac{F_{3}\left(p_{i}\right)}{F_{4}\left(p_{i}\right)} e^{p^{\dddot{\prime}}{ }_{i} t}=B_{p p}+B_{s l} \\
& \phi=\sum \frac{F_{5}\left(p_{i}^{\cdots}\right)}{F_{6}\left(p_{i}^{\cdots}\right)} e^{p^{\dddot{\prime}}{ }_{i}{ }^{t}}=\phi_{p p}+\phi_{s l}
\end{aligned}
$$




\section{Where}

$p_{i}, p_{i}^{\prime \prime}, p_{i}$ is the roots of the characteristic equation,

$H_{p p}, B_{p p}, \Phi_{p p}$ is the simple periodic (forced) components of field strength, induction and flux of quasistationary states,

$H_{s l}, B_{s l}, \Phi_{s l}$ is the free components of magnetic field strength, induction and flux.

For the calculation of forced simple-period components, the imaginary root should be included in equations (18-20) $p=j \omega$, but these components in the work have not been determined; their solutions can be found in [6], [21] in which the propagation of the EM field and the influence of hysteresis in quasi-stationary states are considered. The magnitudes of the free components of field strength, induction and flux can be determined by the numerical method, ie. by developing into sequences [5], [21], [24] according to the formula:

$$
H_{s l}=H_{m} \frac{4}{\Delta_{*}^{2} \cos \alpha} \sum_{k=0}^{\infty}(-1)^{k} \frac{v \omega^{2} c^{2} d \cos 2 v \frac{z_{*}}{\Delta_{*}}}{p_{k}^{2}+\omega^{2}} e^{p_{k} t}
$$

The expected value of the magnetic field strength under the influence of hysteresis and saturation is:

$$
\begin{gathered}
H=H_{p p}+H_{s l}=H_{p p}+H_{m} \frac{4}{\Delta_{*}^{2} \cos \alpha} \sum_{k=0}^{\infty}(-1)^{k} \frac{v \omega^{2} c^{2} d \cos 2 v \frac{z_{*}}{\Delta_{*}}}{p_{k}^{2}+\omega^{2}} e^{p_{k} t} \\
B_{s l}=\mu H_{m}\left[A \sin (\alpha+\psi-\varphi) e^{-\omega \cdot \operatorname{ctg} \alpha \cdot t}+\frac{4}{\Delta_{*}^{2} \cos \alpha} \cdot \sum_{k=0}^{\infty}(-1)^{k} \frac{\left.v \omega^{2} c^{2} d \cos 2 v \frac{z_{*}}{\Delta_{*}}\left(e^{p_{k} t}-e^{-\omega \cdot \operatorname{ctg} \alpha \cdot t}\right)\right]}{p_{k}^{2}+\omega^{2}}\right]
\end{gathered}
$$

The expected value of magnetic induction under the influence of hysteresis and saturation is:

$$
\begin{aligned}
& B=B_{p p}+B_{s l}=B_{p p}+\mu H_{m}\left[A \sin (\alpha+\psi-\varphi) e^{-\omega \cdot \operatorname{ctg} \alpha \cdot t}+\right. \\
& \left.+\frac{4}{\Delta_{*}^{2} \cos \alpha} \sum_{k=0}^{\infty}(-1)^{k} \frac{v \omega^{2} c^{2} d \cos 2 v \frac{z_{*}}{\Delta_{*}}}{p_{k}^{2}+\omega^{2}}\left(e^{p_{k} t}-e^{-\omega \cdot \operatorname{ctg} \alpha \cdot t}\right)\right]
\end{aligned}
$$

The expected value of the flux under the influence of hysteresis and saturation is:

$$
\Phi_{s l}=\sqrt{2} \cdot \mu H_{m} \frac{4}{\Delta_{*}^{2} \cos \alpha} \sum_{k=0}^{\infty}(-1)^{k} \frac{v \omega^{2} c \sqrt{\omega c} d}{\left(p_{k}^{2}+\omega^{2}\right) \sqrt{\left|p_{k}\right|}} e^{p_{k} t}
$$

Where

$$
\begin{aligned}
& A=\frac{\sqrt{\left(\operatorname{ch} 2 m z_{*}+\cos n \Delta_{*}\right)\left(\operatorname{chm} \Delta_{*}+2 n z_{*}\right)}}{\operatorname{chm} \Delta_{*}+\cos n \Delta_{*}}, \Delta_{*}=\frac{\Delta}{a}, z_{*}=\frac{z}{a}, m=\sqrt{1+\sin \alpha}, n=\sqrt{1-\sin \alpha}, \\
& a=\sqrt{\frac{2}{\omega \mu \sigma}} \text { is the depth of penetration of EM waves in sheet, } \\
& c=\cos \alpha+\frac{p_{k}}{\omega} \sin \alpha, d=\cos \varphi-\frac{p_{k}}{\omega} \sin \varphi, v=(2 k+1) \frac{\pi}{2}, p_{k}=-\frac{2 \omega v^{2} \cos \alpha}{\Delta_{*}^{2}+2 v^{2} \sin \alpha}, \operatorname{ch} \sqrt{\frac{\sigma \cdot p}{k_{1} p+k_{2} p}} \frac{\Delta}{2}=0,
\end{aligned}
$$

$p_{k}$ is the characteristic root of the equation. 
Based on the previous relations, that magnetic hysteresis affects the appearance of additional "free" components of field strength, induction and flux in the sheets of the magnetic circuit, where they are damped the slower the wider the hysteresis loops (due to higher value and corresponding time constant value as inverse root values $\tau_{k}=1 / p_{k}$ ).

The derived relations were used to calculate the change in field strength, induction and flux according to the values of relative magnetic permeability. $\mu^{*}=1000$ and $\alpha=250$ which correspond to the electrical steel in the part of the magnetization curve to saturation. The thickness of the sheet metal is $\Delta=0.5 \mathrm{~mm}$, electrical conductivity $\sigma=8 \cdot 10^{6} \mathrm{~S}$ and frequency $f=50 \mathrm{~Hz}$. The dependence of the flux (mean value of induction) on the magnetizing current (field strength on the surface during the transient process) is also given. From the attached diagram in Fig. 1.b it is clearly seen that the three quantities are damped in the transient process in progress $1 / 2$ of period.

\section{The Results of Simulation and Discussion}

Using the electric circuit in Fig. 3, the influence of the saturation effect on the values of currents and voltages in the primary of the transformer in the transient process is simulated. This circuit was applied in the adapted part of the psbxfosaturable.mdl software package MATLAB Simulink [25]. The applied method of verified simulation should confirm the expectations regarding the time forms of developed free components on the linear part and saturation part of selected magnetization characteristic.

In the mentioned program, the saturation characteristics are defined in the block of transformer parameters by linear partial parts of the relation between flux and magnetization current. Remanent flux $p h i_{0}=\Phi_{0}$ suits ot remanent induction $B_{0}=B_{r}$ and point 2 on the saturation characteristic, Fig. 2.b. Saturation characteristic $\{i, p h i\} \equiv\left\{I_{m}, \Phi\right\}$ (per unit: $\mathrm{pu}$ ) in values system starts from $\{0,0\}$.

a)

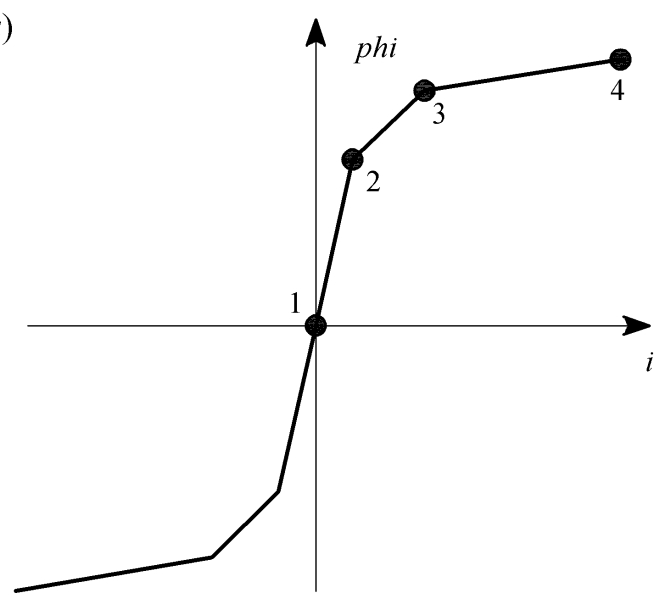

b)

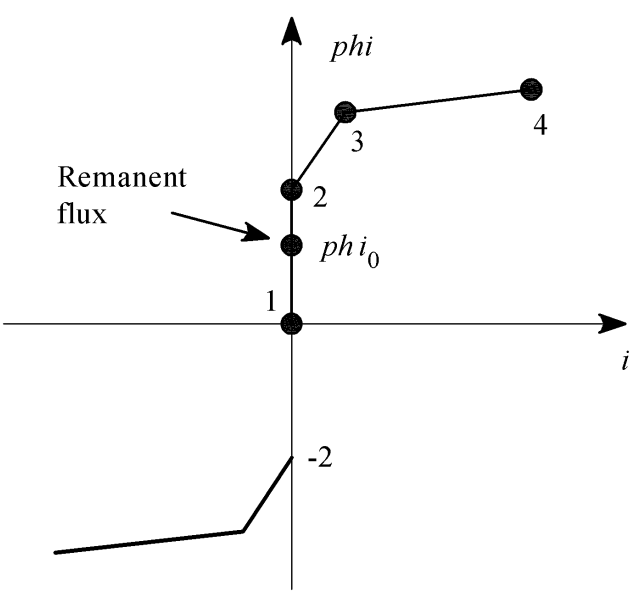

Fig. 2. Transformer magnetic circuit saturation characteristic: a) remanent flux not indicated, b) remanent flux indicated between points 1 and 2

Power System Blockset converts current and flux vectors $\{i, p h i\} \equiv\left\{\vec{I}_{m}(p . u),. \vec{\Phi}(\right.$ p.u. $\left.)\right\}$ into the standard units used in the saturation model Saturable Transformer block:

$$
\begin{aligned}
& \Phi=\Phi_{p u} \Phi_{\text {base }}, I=I_{\text {pu }} I_{\text {base }}, H=H_{p u} H_{\text {base }} \\
& B=B_{\text {pu }} B_{\text {base }}, \Phi_{\text {base }}=\frac{V_{1}}{2 \pi f_{n}} \sqrt{2}, I_{\text {base }}=\frac{P_{n}}{V_{1}} \sqrt{2} \\
& H_{\text {base }}=N_{\text {nav }} I_{\text {base }}=N_{\text {nav }} \frac{P_{n}}{V_{1}} \sqrt{2} \\
& B_{\text {base }}=\frac{\Phi_{\text {base }}}{S_{F e}}=\frac{1}{S_{F e}} \frac{V_{1}}{2 \pi f_{n}} \sqrt{2}
\end{aligned}
$$

Where

$\Phi_{\text {base }}$ is the base flux,

$I_{\text {base }}$ is the base current,

$B_{\text {base }}$ is the induction and base filed strength of maximum value, 
$H_{\text {base }}$ is the amplitudes determined by nominal power, voltage and frequency.

The following values were used in the simulation of the transient process:

- Block Parameters Saturable Transformer,

- Nominal power nad frecuency $\left[P_{n}(\mathrm{VA}) ; f_{n}(\mathrm{~Hz})\right]:[8 ; 50]$,

- Winding 1 parameters $\left[V_{1}(\mathrm{Vrms}) ; R_{1}(\mathrm{pu}) ; L_{1}(\mathrm{pu})\right]:\left[35 \mathrm{e}^{3} / \mathrm{sqrt}(3) ; 0.008 ; 0.032\right]$,

- Winding 2 parameters $\left[V_{1}(\mathrm{Vrms}) ; R_{1}(\mathrm{pu}) ; L_{1}(\mathrm{pu})\right]:\left[35 \mathrm{e}^{3} / \mathrm{sqrt}(3) ; 0.008 ; 0.032\right]$,

- Saturation characteristics $\left[i_{1}(\mathrm{pu}) p h i_{1}(\mathrm{pu}) ; i_{2} \quad p h i_{2}\right.$ (pu)...]: [0 $0 ; 0$ ll.2; 1.0 1.52],

- Core loss resistance and initial flux $\left[R_{m}(\mathrm{pu}) ; p h i_{0}(\mathrm{pu})\right.$ or $\left.\left[R_{m}(\mathrm{pu})\right]: 100\right]$.

In Fig. 3 is a circuit diagram for simulating the effect of magnetic circuit saturation and hysteresis on the values of currents and voltages, field strength, induction and flux during the transient process in a transformer.

Nominal active power losses in the core are included in the adapted part of the psbxfosaturable.mdl software package MATLAB Simulink with equivalent resistance $R_{m}(\mathrm{pu})$. It corresponds to a nominal active power loss of $0.2 \%$ for the nominal voltage $R_{m}=100 \mathrm{pu}$. The initial flux can also be indicated: flux $p h i_{0}(\mathrm{pu})$. If not specified, the initial flux is automatically set in the simulation to the existing start value in the program.

The obtained time forms of electrical components of currents and voltages, and magnetic components of flux induction in the modes of operation on linear part and saturation part of selected magnetization characteristic, confirmed the applicability of the proposed more advanced mathematical model. Also, the obtained results as time forms can be converted into numerical data that would be a valuable aid in design of magnetic circuits of power transformers.

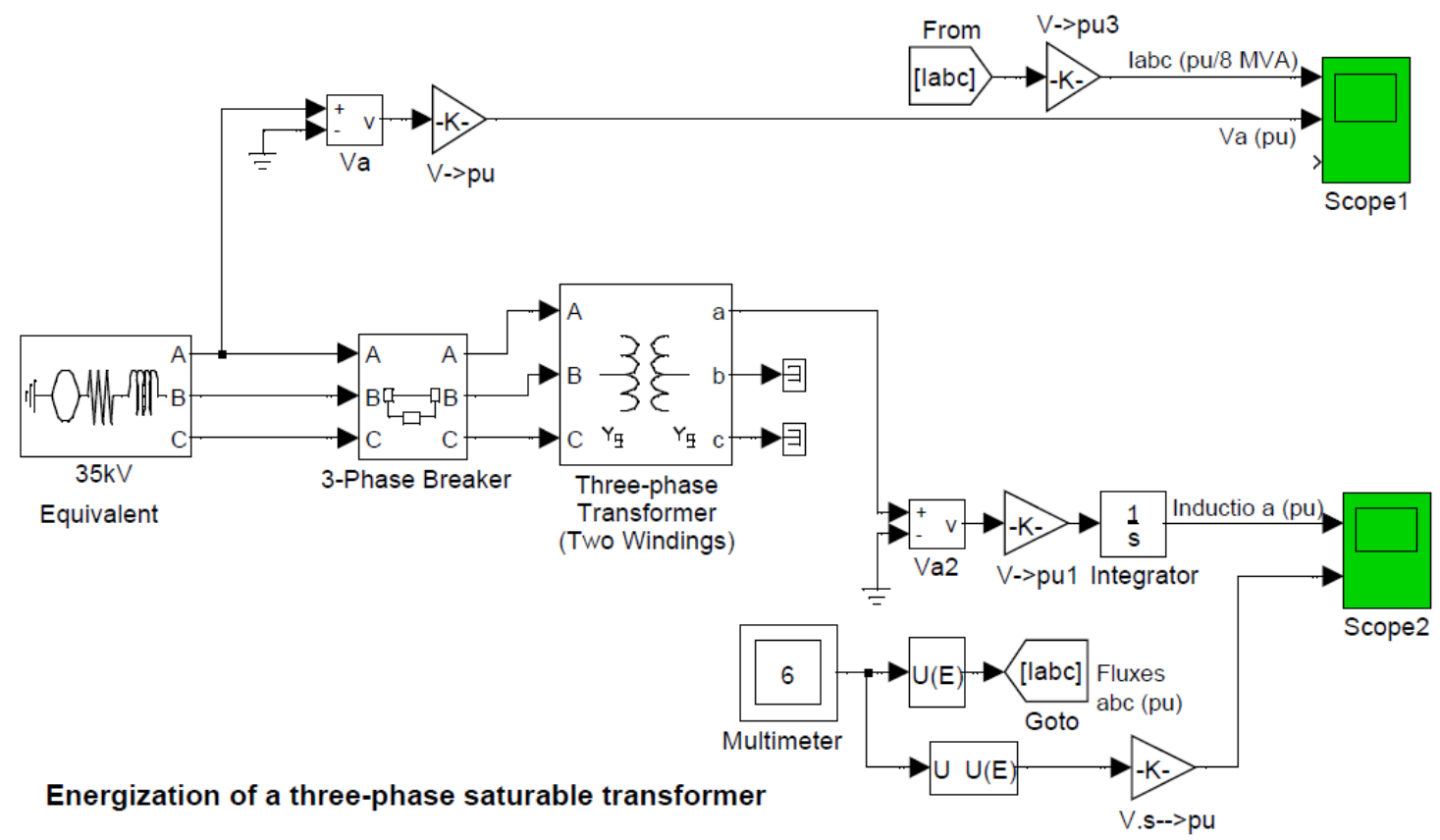

Fig. 3. Circuit diagram for simulating the effect of magnetic circuit saturation and hysteresis

Example: Three-phase transformer $8 \mathrm{MVA}, 35 / 10 \mathrm{kV}$ YY switched on into net $35 \mathrm{kV}$. Active loss in magnetic (core): 100 pu. Saturation characteristic: [0 0; 0 1.2; 1.0 1.52]. Remanent flux $0.6 \mathrm{pu}$.

In this circuit, the magnetization currents of the individual phases are calculated and simulated by integrating the primary currents; phase A voltage is calculated and simulated by integrating the primary phase A voltage.

Magnetic induction of phase A and fluxes generated by magnetization currents of individual phases were obtained by using measuring equipment Multimeter block.

In Fig. 4 shows the obtained simulation results for magnetization currents, voltages, magnetic induction and magnetic circuit flux with the assumed magnetization characteristic and dimensions at sinusoidal change of voltage, ie induction which depends on its value.

The part related to the initial part of the transient process, lasting 0.05 seconds, is clearly recognized, and the influence of the saturation curve of the magnetization curve of the magnetic circuit can be seen on the diagrams showing the induction in the selected phase (a) and the flux in all three phases (a, b, s). From the shape of these 
diagrams showing the induction in phase (a) it is not difficult to conclude that a strong aperiodic component is present in this component which is announced in equation (24), and something similar is confirmed by equation (25).
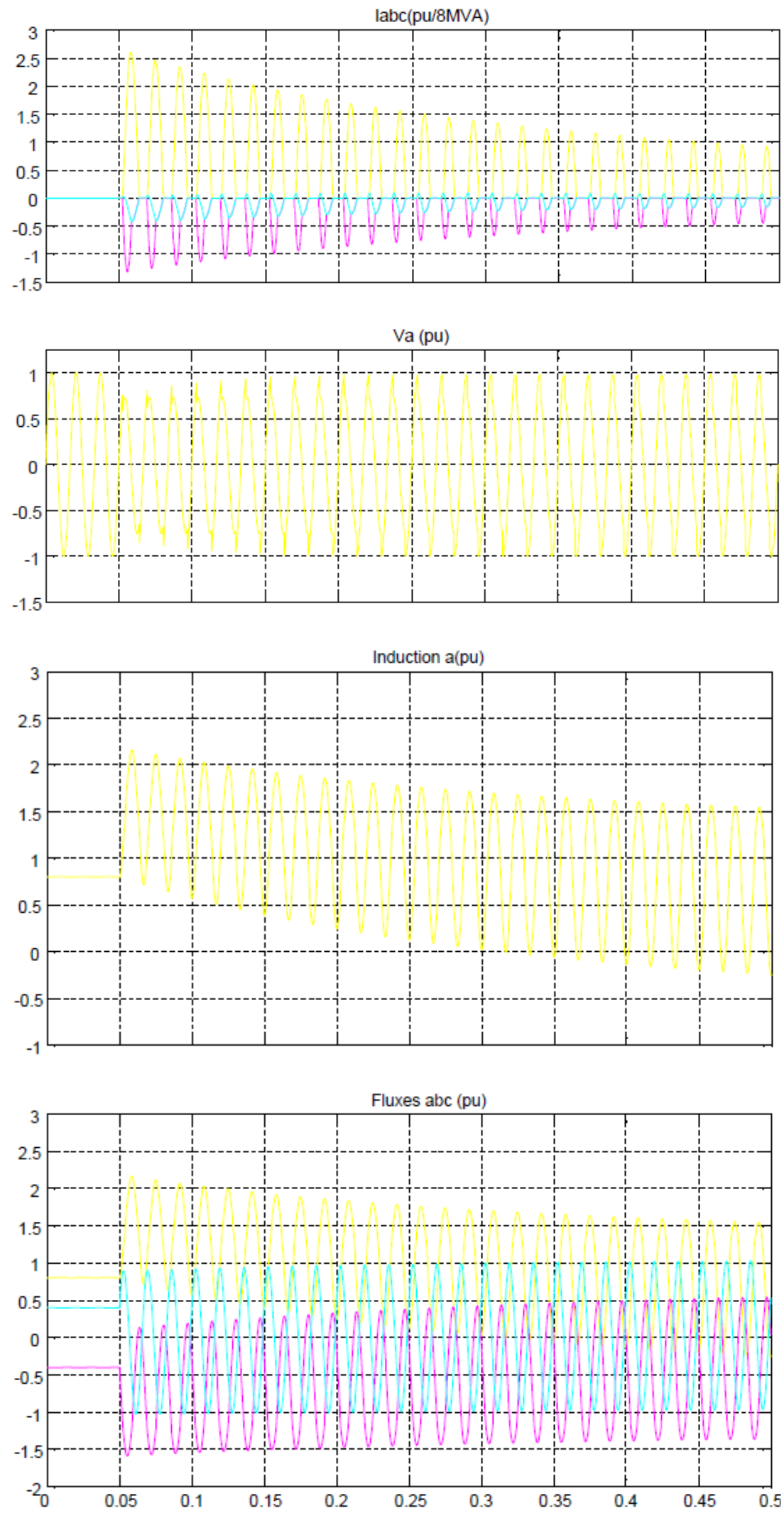

Fig. 4. Results of simulation of transient process in power transformer under the influence of saturation and hysteresis in magnetic circuit 


\section{Conclusion}

The paper develops a theory for combined EM parameters of a magnetic circuit, which is equally important for rotating machines and transformers, so simulation has been performed on the example of a magnetic circuit of transformer (the same sheets exists in electric machine circuits). Sheets in magnetic circuits and transient processes were affected by hysteresis.

The considered analytical method for calculation of transient process on linear part of the hysteresis loop is applicable to all devices which magnetic circuits work on the linear part of magnetization characteristic and enables the determination of free components of field strength, induction and flux.

The mathematical model refers to assumed magnetization curve and dynamic magnetization process, but it must be known that sheet characteristic can be different and special in each construction in the magnetic circuit and depends on sheet thickness and EM field frequency, which means that there are no universal characteristics magnetization that will be applied in all magnetic circuits. Therefore, instead of universal magnetization characteristics, it is more expedient to form and use "special" characteristics obtained in conditions that are close to the operating conditions of a particular magnetic circuit in a device that can be easily simulated in one of the software packages such as MATLAB Simulink. In this way, the scope of required measurements can be significantly reduced and the accuracy of methods for calculating designed device can be increased.

Using theoretical model and adapted psbxfosaturable.mdl software package MATLAB Simulink it has been confirmed that hysteresis affects increase of time constants of free components and slows down and dampens the transient processes in magnetic circuits. The results obtained as time forms of currents, voltages, magnetic induction and magnetic flux can be a valuable aid in the design of magnetic circuits of power transformers.

\section{References}

[1] P. Spalević, B. Mirić, D. Vučković, S. Panić, "Magnetic field of inductive loop-theory and experimental results", Elektronika Ir Elektrotechnika, Vol. 99, Issue 3, 2010, pp. 83-88.

[2] N. Marković, S. Bjelić, J. Živanić, Z. Bogićević, "Analysis and Estimation of Values of Currents and Voltages at the Disturbances in Induction Machine Using Tested Matlab Simulation”, International Journal of Intelligent Systems and Applications, IJISA 2014, Vol. 7, No. 1, pp. 1-8, DOI: 10.5815/ijisa.2015.01.01.

[3] M. Mišić, Z. Bogićević, S Bjelić, "Electrodynamic Forces between Electrical Conductors and Cylindrical Magnetic Shields", British Journal of Applied Science \& Technology, Article no. BJAST.20541, 11(4), 2015, pp. 1-11, DOI: 10.9734/BJAST/2015/20541.

[4] S. Bjelić, Z. Bogićević, "Computer Simulation of Theoretical Model of Electromagnetic Transient Processes in Power Transformers”, International Journal of Information Technology and Computer Science, IJITCS 2014, Vol. 6, No. 1, pp. 1-12, DOI: $10.5815 /$ ijitcs.2014.01.016.

[5] B. Geller, A. Veverka, "Импулсные процессы в электрических машины”, Энергия, Москва, 1973.

[6] J.M. Živanić, V. Ostraćanin, Lj. Filipović, "Electric machines 3”, Technical Faculty, Čačak, 2010.

[7] N. Watson, J. Arillaga, "Power Systems Electromagnetic Transients Simulation the Institution of Engineering and Technology", IET Power and Enegy series 39, 2003, pp. 160-181.

[8] J.R. Linders, "Electric wave distorsion", Their hidden costs and containment IEEE Trans. Ind. Appl. 15, No. 5, 1979, pp. 458474.

[9] A.E. Fitzegerald, C. Kingsley, "Electric Machinery”, Fifth Edition, McGraw-Hill Publishing Company, New York, 1990.

[10] J. Surutka, "Electromagnetics", Academic Thought (Planeta print), Belgrade, 2006.

[11] А.И. Вольдек, Электрические машины, Ленинградское отделение “Энергия”, 1974.

[12] L.W. Match, J.D. Morgan, "Electromagnetic and Electromechanical Machines", Third Edition, Harper\&Row Publishers, New York, 1986.

[13] S.J. Chapman, "Electric Machinery Fundamentals", Third Edition, McGraw Hill, Boston, 1999.

[14] N. Marković, S. Bjelić, J. Živanić, U. Jakšić, "Simulation of the Impact of Higher Harmonics on the Transient Process of Induction Machine Fed From PWM Inverters", Preliminary communication, Technical Gazette, DOI: 10.17559/TV20150502231618, Vol. 24, No. 1, 2017, pp. 265-271.

[15] N. Marković, S. Bjelić, J. Živanić, V. Milićević, Z. Milićević, "Model of Transient Process Where Three-Phase Transducer Feeds Induction Motor Equivalented as a Variable Active-Inductive Load", Research Article, Mathematical Problems in Engineering, Vol. 2016, Article ID 6740261, http://dx.doi.org/10.1155/2016/6740261, 2016, 14 pages.

[16] E.F. Fuchs, M. Poloujadof, G.W. Neal, "Starting performance of saturable three-phase induction motor's", IEEE Transactions on Energy Conversion, Vol. EC-3, No. 3, 1998, pp. 624-635.

[17] Z. Bogićević, N. Marković, P. Spalević, M. Vujičić, "Development of the algorithm for calculation of transformer magnetic circuit”, ICDQM-2015 (International Conference Dependability and Quality Management), Belgrade, Serbia, 2015, pp. 270277.

[18] B. Wangsilabatra, S. Tunyasrirut, W. Permpoonsinsup, "Three Phase Induction Motor Drive Using Hybrid Fuzzy PI Controller Based on Field Oriented Control”, I.J. Image, Graphics and Signal Processing, IJIGSP 2018, Vol. 10, No. 1, pp. 1-10, DOI: 10.5815/ijigsp.2018.01.01.

[19] M.A. Rozenblat, "Magnitnie elementi avtomatiki i vičislitelnoi tehniki”, Nauka, Moskva, pp. 31-41, 78-85, 1978. 
[20] A.A. Mahfouz, M.K. Mohammed, F.A. Salem, "Modeling, Simulation and Dynamics Analysis Issues of Electric Motor, for Mechatronics Applications, Using Different Approaches and Verification by MATLAB/Simulink", International Journal of Intelligent Systems and Applications, IJISA 2013, Vol. 5, No. 5, pp. 39-57, DOI: 10.5815/ijisa.2013.05.06.

[21] M.L. Levinštejn, "Operatorovy počet v elektrotechnice", SNTL, Praha, 1977.

[22] Lou van der Sluis, "Transients in Power Systems", John Wiley \& Sons Ltd, 2001.

[23] J. Kulda, "Magneticke pole v silnoproude elektrotechnice", ACADEMIA, Praha, pp. 44-46, 78-80, 1974.

[24] И.Н. Бронштеин, К.А. Семендјајев, “Справочник по математике дја инжињеров и учащихся втузов”, Физикоматематическаја литература, Москва, 1962.

[25] MATLAB SIMULINK Sim Power System, Copyright 1984-2002 The Math Works, Version 6.5.0,180913a, June 2, 2000.

\section{Authors' Profiles}

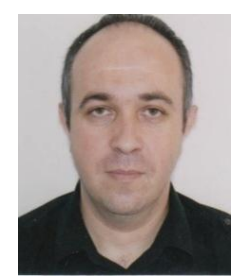

Nenad A. Marković: Professor at study programs Mechanical engineering and Road Traffic on Kosovo and Metohija Academy of Applied Studies, Department Urosevac - Leposavic. He is received his Ph.D. degree from Faculty of technical Sciences in Cacak-University of Kragujevac. Author and co-author is of many textbooks, exercises and over 125 papers published in international and Serbian journals.

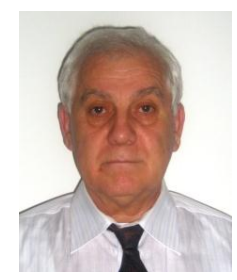

Slobodan N. Bjelić: Full-time professor at Faculty of Technical Sciences University in Pristina with temporarily seat seat in Kosovska Mitrovica. He received his Ph.D. degree in 1982, field of interest: Electrical EngineeringComputer Science-Protective Relays-Electrical Installation and Illumination Engineerings-Electrical Power Converters. He is author and co-author of 10 textbooks and over 200 scientific and professional papers published in international and Serbian journals.

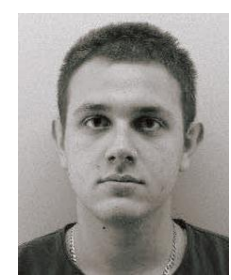

Filip N. Marković: Student on $4^{\text {rd }}$ year at Faculty of Technical Sciences-University in Pristina with temporarily seat in Kosovska Mitrovica. Field of interest: Electrical and Computer Engineering, Computer Engineering and Software Engineering. He is author and co-author of several papers in the field of electrical engineering and computer science published in national and international peer-reviewed journals.

How to cite this paper: Nenad A. Marković, Slobodan N. Bjelić, Filip N. Marković, " The Influence of Magnetic Hysteresis in Magnetic Circuit Sheets on Transient Processes in Electrical Machines", International Journal of Image, Graphics and Signal Processing(IJIGSP), Vol.13, No.3, pp. 1-12, 2021.DOI: 10.5815/ijigsp.2021.03.01 